\title{
Manifesto de Natal - Por uma política afirmativa no recrutamento, formação, provimento, retenção e suporte para as médicas de família em contexto rural e remoto
}

Natal Manifest - For an affirmative policy in the establishment, recruitment, continuing education and support to the female family doctors in rural and remote contexts

\section{Manifiesto de Natal - Por una política comprometida en la radicación, contratación, formación continuada y apoyo para las médicas de familia em contextos rurales y remotos}

Monica Correia Lima. Prefeitura Municipal de Cajati (Vale do Ribeira). Cajati, SP, Brasil. E-mail: monicalima@usp.br (Autora correspondente)

Marcela Cuadrado. Universidad de la Republica del Uruguay (UDELAR). Montevideo, Uruguai. E-mail: marcelacua@gmail.com Thereza Cristina Gomes Horta. Centro Universitário de Belo Horizonte (UniBH); Associação Beneficente dos Empregados da Cia Sid Belgo Mineira (ABEB). Belo Horizonte, MG, Brasil. E-mail: thehorta@gmail.com Magda Almeida. Universidade Federal do Ceará (UFC). Fortaleza, CE, Brasil. E-mail: magnhets@gmail.com Ariane Pinheiro. Prefeitura Municipal de João Monlevade. João Monlevade, MG, Brasil. E-mail: arianepinheiro@yahoo.com.br Paula Christianne Gomes G. Souto Maia. Universidade Federal de Campina Grande (UFCG). Cajazeiras, PB, Brasil.

E-mail: pcggsm@gmail.com

Candida Pereira Melo. Secretaria Municipal de Saúde de São Leopoldo. São Leopoldo, RS, Brasil. E-mail: candida.melo@furg.br Eneline de Andrade Heráclito Gouveia Pessoa. Centro Universitário Cesmac (CESMAC). Maceió, AL, Brasil.

E-mail: eneline.gouveia@gmail.com

Viviane Xavier. Universidade Federal de Pernambuco (UFPE). Recife, PE, Brasil. E-mail: vivianexavier09@gmail.com Maria Carolina Pereira da Rocha. Pontifícia Universidade Católica de São Paulo. São Paulo, SP, Brasil.

E-mail: mcarolrocha@ hotmail.com

\section{Resumo}

O presente documento foi produzido com base na oficina de Gênero sobre o trabalho das Médicas de Família em áreas rurais e remotas, durante o $13^{\circ}$ Congresso Brasileiro de Medicina de Família e Comunidade, de 8 a 12 de julho de 2015. Objetivou o estabelecimento de importantes pressupostos para o recrutamento, formação, provimento, retenção e suporte para o trabalho das médicas de família e comunidade nas mais diversas áreas rurais, rururbanas e remotas do país, em seus mais diversos cenários, devendo servir de base para estudos e formulação de políticas públicas sobre tais aspectos.
Palavras-chave:

Medicina de Família e Comunidade Identidade de Gênero Saúde da População Rural

Recursos Humanos em Saúde
Como citar: Lima MC, Cuadrado M, Horta TCG, Almeida M, Pinheiro A, Maia PCGGS, et al. Manifesto de Natal - Por uma política afirmativa no recrutamento, formação, provimento, retenção e suporte para as médicas de família em contexto rural e remoto. Rev Bras Med Fam Comunidade. 2016;11(38):1-5. http://dx.doi.org/10.5712/rbmfc11(38)1247
Fonte de financiamento: declaram não haver. Parecer CEP: não se aplica. Conflito de interesses: declaram não haver. Procedência e revisão por pares: revisado por pares. Recebido em: 22/11/2015 Aprovado em: 29/03/2016. 


\section{Abstract}

This document was produced on the basis of the Gender workshop on the work of female Family Doctors in rural and remote areas, during the $13^{\text {th }}$ Brazilian Congress of Family and Community Medicine, from July 8-12, 2015. It aimed to establish important assumptions for the recruitment, training, provision, and support for the work of female family physicians in the many rural, rururban and remote areas of the country, in its various different scenarios, and should serve as a basis for studies and formulation of public policies on such aspects.

\section{Resumen}

El presente documento fue producido con base en el taller de Género sobre el trabajo de las Médicas de Familia (Cabecera) en áreas rurales y remotas, durante el $13^{\circ}$ Congreso Brasileño de Medicina de Familia y Comunidad, de 8 a 12 de julio de 2015. Su objetivo es el estabelecimiento de importantes presuposiciones para el reclutamiento, formación, provisión, fijación y suporte para el trabajo de las médicas de familia y comunidad en las más distintas áreas rurales, rururbanas y remotas del país bajo los más diversos escenarios, debiendo servir de base para estudios y formulación de políticas públicas sobre tales aspectos.

\section{Keywords:}

Family Practice

Gender Identity

Rural Health

Health Manpower

Palabras clave:

Medicina Familiar

y Comunitaria

Identidad de Género

Salud Rural

Recursos Humanos en Salud

Nós, médicas de família do Grupo de Trabalho de Medicina Rural, presentes na oficina de Gênero em Medicina de Família Rural, durante o 13ํㅡㄹ Congresso Brasileiro de Medicina de Família e Comunidade, de 8 a 12 de julho de 2015, entendemos ser urgente e necessário pensar nas diferenças de gênero no recrutamento, formação, provimento, retenção e suporte para as médicas de família e comunidade nas mais diversas áreas rurais, rururbanas e remotas, assim como em cenários específicos, como assentamentos, comunidades isoladas, quilombos, caiçaras, ciganas, ribeirinhas, indígenas e tantas quantas existirem pela diversidade populacional de tamanho continental como este Brasil possui.

Consubstanciadas por documentos internacionais que afirmam a necessidade de inserir médicas no meio rural tendo em vista suas características peculiares de trabalho que se somam às características dos médicos (sendo então ambos primordiais nesse meio), ${ }^{1,2}$ entendemos que há pouca pesquisa em nível nacional que tipifique a força de trabalho do gênero feminino, servindo como subsídio para a formulação de políticas afirmativas para inserção e manutenção das médicas em campo rural.

Precisamos considerar que o contingente de mulheres nas universidades médicas brasileiras tem aumentado: em 1990, as médicas atuantes nas regiões Norte, Nordeste, Sul, Sudeste e Centro-Oeste representavam, respectivamente, 34\%, 38\%, 25\%, 31\% e 23\% do total. Dez anos depois, houve aumento desse percentual em todas as regiões para $41 \%, 43 \%, 35 \%, 41 \%$ e $37 \%$, respectivamente, representando no total $40 \%$ da força de trabalho médico no Brasil. ${ }^{3}$

Dados do Conselho Federal de Medicina (CFM) de 2015 indicam que 39\% das médicas ativas atuam fora das capitais dos estados. A região Sul chega a ter $50 \%$ das suas médicas inscritas no interior (Tabela 1), enquanto apenas $20 \%$ das médicas das regiões Norte e Nordeste moram fora das capitais, apesar de representarem mais de $40 \%$ da força de trabalho médico dessas áreas.

Tabela 1. Distribuição das médicas ativas por região e local de inscrição do Conselho Federal de Medicina, 2015*.

\begin{tabular}{lccccc}
\hline & Capital & Interior & Número de Médicas ativas & $\%$ médicas & $\%$ médicas no interior \\
\hline CO & 8773 & 3751 & 12524 & $41 \%$ & $30 \%$ \\
N & 24625 & 6804 & 31429 & $45 \%$ & $22 \%$ \\
NE & 5981 & 1484 & 7465 & $43 \%$ & $20 \%$ \\
SU & 54849 & 44415 & 99264 & $44 \%$ & $45 \%$ \\
S & 11833 & 11937 & 23770 & $39 \%$ & $50 \%$ \\
\hline
\end{tabular}

CO: Centro-Oeste; N: Norte; NE: Nordeste; SU: Sudeste; S: Sul. Fonte: http://portal.cfm.org.br/index.php?option=com_estatistica. ${ }^{*}$ excluídas as categorias "médicos de outros estados" e "desatualizados" por não indicarem a localização. 
Dessa forma, sendo de tamanha urgência, o grupo de trabalho que iniciou suas discussões na oficina de gênero no trabalho das médicas de família em áreas rurais, cujo objetivo geral foi construir em conjunto a base para uma proposta política para a formação, retenção e trabalho de médicas de família e comunidade para as áreas rurais e remotas do país, faz então as seguintes propostas a partir dos objetivos específicos:

- Propor medidas para o aumento de mulheres médicas de família e comunidade em áreas rurais e remotas:

- Que haja recrutamento de estudantes do sexo feminino provenientes de áreas rurais para a medicina.

- Que a inserção da aluna de medicina no ambiente rural e remoto seja precoce, de forma sistematizada, em redes de intercâmbio, para que ela possa ter experiências de vivência com projetos de intervenção em vários cenários.

- Que a discussão sobre as questões de gênero seja presente e consistente na graduação e na pós-graduação, permitindo ir além do caráter reducionista que aprisiona o debate apenas dentro do campo da sexualidade.

- Que a inserção do residente do primeiro ano (R1) da nova apresentação da residência médica seja também em áreas rurais e remotas em seus diversos cenários e que haja expansão do R3 rurais para todas as residências médicas em medicina de família e comunidade.

- Que existam políticas municipais/estaduais e federais levando em conta essas diferenças, a fim de que o trabalho em áreas rurais e remotas seja um atrativo para o acadêmico, e não uma ação de última escolha.

- Que sejam consideradas e avaliadas as expertises de outros países ${ }^{4,5}$ no desenvolvimento de programas de formação para medicina em áreas rurais, executando políticas alicerçadas na melhor evidência existente, sem desconsiderar a necessária contextualização.

- Formular medidas de suporte ao trabalho das médicas de família em áreas rurais e remotas do país:

- Que, apesar das diferentes modalidades de contratação, seja garantida à mulher a licença maternidade de seis meses, para que não haja redução salarial durante seu afastamento e para que ela possa estar com a sua prole no período recomendado para o aleitamento materno exclusivo.

- Que haja a flexibilização da carga horária da mulher médica durante períodos de seu ciclo vital em que sua presença é fundamental como mãe e que se estende para além dos seis primeiros meses.

- Que nesses períodos a mulher também possa contar com a presença do cônjuge por meio de leis que possibilitem, por exemplo, a ampliação da licença parental ou com a licença compartilhada.

- Que haja flexibilização e adequação de estrutura para a formação continuada da médica de família nessas áreas, bem como uma rede de apoio acadêmico e de trabalho, a fim de que a profissional não permaneça com sensação de isolamento. 
- Que haja estrutura para o seu trabalho desde aspectos ligados a transporte, segurança, equipamentos, qualidade da equipe.

- Construir em conjunto propostas para retenção de médicas de família e comunidade em áreas rurais:

- Que haja equiparação salarial entre o trabalho dos médicos e médicas de família em áreas rurais (onde há discrepância), havendo estabilidade de vínculo, e aplicação de todos os benefícios já garantidos por lei para o trabalho feminino, bem como a construção de políticas afirmativas para execução do trabalho da mulher levando em conta sua dupla e até tripla jornada de trabalho.

- Que haja a possibilidade de remuneração extra por exercício da medicina fora do seu horário de trabalho.

- Que seja levada em conta a necessidade de fixar o seu companheiro em termos de trabalho.

- Que haja estrutura para o suporte de sua família enquanto a mulher médica/mãe cuida da saúde da comunidade, creche, escola de qualidade, opções de lazer.

- Que a população adscrita do trabalho da médica mulher seja reduzida, levando em conta suas habilidades (já comprovadas por trabalhos que se referem ao perfil da médica mulher).

- Garantir que as médicas de família e comunidade em áreas rurais tenham treinamento de habilidades específico para as áreas onde atuam ou atuarão:

- Que haja treinamento disponível em horários flexíveis.

- Que o treinamento possa ser realizado por professoras/preceptoras (sexo feminino).

- Que haja desenvolvimento adequado de habilidades em áreas importantes para a prática de medicina rural, incluindo emergência, anestesiologia, habilidades cirúrgicas e obstétricas.

- Que a educação médica continuada (EMC) seja acessível em seu custo, localização, retaguarda para as crianças (se for o caso) e carga horária. Se possível, realizar EMC durante o horário de trabalho.

- Que haja programas de retreinamento para mulheres que queiram transitar de seus empregos de meio horário para carga horária completa ou que retornarão ao trabalho após pausa para cuidar dos filhos.

- Que haja treinamento para habilidades de negociação, administração e liderança.

- Reconhecer a sub-representação das mulheres em posições de tomada de decisão (e em outras posições) e garantir representação equiparada de mulheres e homens em organizações profissionais para médicos de família em áreas rurais.

Enfim, a múltipla função do médico de família em áreas rurais, somada à tripla carga das mulheres médicas, requer que de modo sensível e estratégico haja uma política voltada para essa captação, formação, fixação e educação continuada dessa profissional, a fim de que possa expressar equilíbrio, humanidade, compaixão e amor pela arte da medicina de família em áreas rurais e remotas. 


\section{Referências}

1. WONCA Working Party on Rural Practice. Políticas para Médicas de Família em Áreas rurais. Monash University School of Rural Health: Traralgon, Vic. (tradução gt de medicina de família rural da SBMFC). 2002. [Citado 18 Jul 2016]. Disponível em: https://docs.google.com/viewer?a=v\&pid=sites\&srcid=ZGVmYXVsdGRvbWFpbnxndG1IZGljaW5hcnVyYWx8Z3g6 NDk1NWY3ZWU2YjZiZDMw

2. Wainer J. Women and rural medical practice: the importance of doing it differently in the 21 st century. WONCA Rural Medical Education Guidebook. WONCA, 2015 [Citado 18 Jul 2016]. Disponível em: http://www.globalfamilydoctor.com/ groups/WorkingParties/RuralPractice/ruralguidebook.aspx

3. Brasil. Ministério da Saúde/SGTES/DEGERS/CONPROF - conselhos profissionais. [Citado 18 Jul 2016]. Disponível em http://tabnet.datasus.gov.br/cgi/deftohtm.exe?idb2011/e01.def

4. Ellsbury KE, Baldwin LM, Johnson KE, Runyan SJ, Hart LG. Gender-related factors in the recruitment of physicians to the rural Northwest. J Am Board Fam Pract. 2002;15(5):391-400.

5. Ozolins IZ, Greenwood G, Beilby J. Keeping women doctors in the country. Rural and Remote Health 4: 268. (Online) 2004 [Citado 18 Jul 2016]. Disponível em: http://www.rrh.org.au/articles/showarticlenew.asp?ArticlelD=268 